\title{
Thèse remarquée
}

\author{
Discrimination des effets chimiotoxiques et radiotoxiques de l'uranium : définition de \\ marqueurs biologiques pour l'évaluation des risques professionnels dans \\ l'industrie nucléaire. Carine Darolles. Thèse de l'université Aix-Marseille II, Faculté \\ de Pharmacie, École doctorale des sciences de l'environnement (ED251), soutenue le \\ 6 mai 2010. Laboratoire d'accueil : Laboratoire de radiotoxicologie expérimentale, \\ IRSN/DRPH/SRBE, Pierrelatte. Tuteur IRSN : Fabrice Petitot. Directeur de thèse : \\ Pr Michel De Meo, université d'Aix Marseille II.
}

La toxicité de l'uranium résulte de la combinaison de ses propriétés chimiques, en tant que métal lourd, et de ses propriétés radiologiques, en tant qu'émetteur de rayonnements ionisants (principalement rayonnements alpha). L'identification de marqueurs biologiques permettant de discriminer pour un composé uranifère donné la part de sa toxicité chimique de la part de sa toxicité radiologique évitera de sous-estimer les effets sur la santé des mélanges isotopiques ayant une faible activité spécifique et donc un faible impact en termes de dose tels que l'uranium appauvri en ${ }^{235} \mathrm{U}$. Les données de la littérature montrent que les meilleurs candidats pour répondre à cette problématique sont des marqueurs cytogénétiques.

La recherche de biomarqueurs de contamination interne par l'uranium a été mise en œuvre sur trois modèles cellulaires exposés in vitro (fibroblastes de souris, Darolles et al., 2010a ; lymphocytes humains, Darolles et al., 2010b) ou in vivo (lymphocytes de rat) à différents mélanges isotopiques de l'uranium. Les plans expérimentaux de contamination définis ont permis de dissocier l'influence respective des 2 facteurs, effet chimique et effet radiologique de l'uranium, sur les variables cytogénétiques mesurées en combinant dans un schéma factoriel à différents niveaux d'exposition à l'effet chimique (concentration) à différents niveaux d'exposition à l'effet radiologique (activité). Le test des micronoyaux couplé à une hybridation in situ de sondes pancentromériques a ensuite été réalisé sur les cellules ainsi exposées.

Ces études révèlent que le seul dénombrement des cellules binucléées avec un micronoyau ne suffit pas à évaluer avec précision la génotoxicité de l'uranium. En revanche, les fréquences d'apparition de cellules binucléées avec un micronoyau centromère négatif ou avec un pont nucléoplasmique peuvent refléter le niveau des effets radiotoxiques d'un mélange isotopique donné de l'uranium. Par ailleurs, la numération des cellules binucléées avec un micronoyau centromère positif et des cellules mononucléés avec des micronoyaux peuvent refléter le niveau des effets chimiotoxiques d'un mélange isotopique donné de l'uranium.

Ces marqueurs cytogénétiques validés sur différents modèles biologiques permettent de déterminer pour un mélange isotopique donné de l'uranium (i.e. : un enrichissement en ${ }^{235} \mathrm{U}$ donné) la part des effets génotoxiques liée à sa chimiotoxicité de la part des effets génotoxiques liée à sa radiotoxicité. Ces marqueurs biologiques pourraient compléter les marqueurs classiques de dosimétrie pour évaluer les conséquences de la contamination interne à l'uranium. 


\section{THÈSE REMARQUÉE}

Ces résultats prometteurs devront être validés sur des prélèvements de sang issus de travailleurs du nucléaire ayant subi une contamination interne accidentelle à l'uranium.

Darolles C., Broggio D., Feugier A., Frelon S., Dublineau I., De Meo M., Petitot F. (2010a) Different genotoxic profiles between depleted and enriched uranium, Toxicol. Lett. 192, 337 348.

Darolles C., Broggio D., Feugier A., De Meo M., Petitot F. (2010b) Discrimination of radiotoxic and chemotoxic effects of uranium with the micronucleus centromere assay, soumis à publication. 